\title{
Fundamentals of Implant Dentistry Volume II: Surgical Principles
}

Authors: Peter K. Moy / Alessandro Pozzi / John Beumer III

Publisher: Quintessence Publishing

Language: English

ISBN: 978-0-86715-584-6

Edition: 1/e

Publish Year: 2017

Pages: 448, illustrated

Price: $168.00 €$

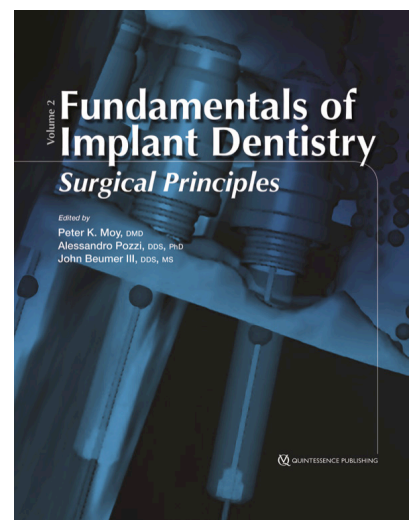

The second volume of the book entitled "Fundamentals of Implant Dentistry" tackles the importance of an interdisciplinary approach and demonstrates how the surgeon plays a leading role during treatment planning and surgical management.

The book has 13 chapters. The first four chapters speak about the basic principles of oral implantology, from the patient's medical history to the evolution of modern dental implant and the interdisciplinary workup. The next seven chapters illustrate the basic surgical procedure used in implant dentistry, tilted and zygomatic implant, hard and soft tissue grafting, reconstruction of major defects with implants and surgical consideration of the esthetic zone and various loading protocols. The last two chapters tackle complication and follow up. The success rate of dental implants is influenced by the patient's adequate home care.

This book teaches the clinicians providing implant treatment the importance of a good surgical and prosthetic techniques and maintenance and follow up. The authors present new concept techniques and materials introduced to the field in oral implantology to provide the practitioner with success in his daily activity.

DOI: 10.25241/stomaeduj.2017.4(2).bookreview.3

Florin-Eugen

Constantinescu

DDS, PhDStudent 